\title{
Antioxidant Effects of Irisin in Liver Diseases: Mechanistic Insights
}

\author{
Junzhou Zhao $\mathbb{D}^{1,2}$ Linlan Qiao, ${ }^{1,2}$ Jian Dong, ${ }^{1,2}$ and Rongqian $\mathrm{Wu}^{1}$ \\ ${ }^{1}$ National Local Joint Engineering Research Center for Precision Surgery \& Regenerative Medicine, Shaanxi Provincial Center for \\ Regenerative Medicine and Surgical Engineering, First Affiliated Hospital of Xi'an Jiaotong University, Xi'an, \\ Shaanxi Province, China \\ ${ }^{2}$ Department of Hepatobiliary Surgery, First Affiliated Hospital of Xi'an Jiaotong University, Xi'an, Shaanxi Province, China
}

Correspondence should be addressed to Rongqian Wu; rwu001@mail.xjtu.edu.cn

Received 17 September 2021; Revised 19 November 2021; Accepted 10 December 2021; Published 7 January 2022

Academic Editor: Erika Ramos-Tovar

Copyright (c) 2022 Junzhou Zhao et al. This is an open access article distributed under the Creative Commons Attribution License, which permits unrestricted use, distribution, and reproduction in any medium, provided the original work is properly cited.

Oxidative stress is a crucial factor in the development of various liver diseases. Irisin, a metabolic hormone discovered in 2012, is mainly produced by proteolytic cleavage of fibronectin type III domain containing 5 (FNDC5) in skeletal muscles. Irisin is induced by physical exercise, and a rapidly growing body of literature suggests that irisin is, at least partially, responsible for the beneficial effects of regular exercise. The major biological function of irisin is believed to be involved in the maintenance of metabolic homeostasis. However, recent studies have suggested the therapeutic potential of irisin against a variety of liver diseases involving its antioxidative function. In this review, we aim to summarize the accumulating evidence demonstrating the antioxidative effects of irisin in liver diseases, with an emphasis on the current understanding of the potential molecular mechanisms.

\section{Introduction}

Irisin is a metabolic hormone discovered by Spiegelman's group in 2012 [1]. It consists of 112 amino acid residues and is mainly produced by cleavage of fibronectin type III domain containing 5 (FNDC5) in response to exercise. A rapidly growing body of literature suggests that irisin is at least partially responsible for the beneficial effects of regular exercise, such as weight loss, stronger bones, and better cardiovascular health $[2,3]$. The major biological function of irisin is believed to be involved in the maintenance of metabolic homeostasis. In adipose tissues, irisin induces browning of white adipose tissue to increase energy expenditure [4]. In myocytes, irisin improves fatty acid oxidation and glucose utilization [5]. In hepatocytes, irisin suppresses gluconeogenesis, lipogenesis, and lipid accumulation [6, 7]. However, recent studies have suggested that irisin has potent antioxidative properties and is expected to be a potential therapeutic agent to protect tissues from oxidative stress-induced injury [8]. Oxidative stress occurs when the production of free radicals overwhelms the antioxidant system. The imbalance between oxidants and anti- oxidants can lead to structural and functional damage in the liver [9]. Increased oxidative stress is the underlying mechanism of many liver diseases, regardless of the cause of the liver disorder [10]. Oxidative stress is a crucial factor in the development of various liver diseases, including nonalcoholic fatty liver disease (NAFLD), hepatic ischemia reperfusion injury (HIR), and liver cirrhosis (Figure 1). Considering the global burden of liver diseases, herein, we mainly discuss the antioxidative effects of irisin in the liver. We aim to summarize the accumulating evidence demonstrating the antioxidative effects of irisin in liver diseases, with an emphasis on the current understanding of the potential molecular mechanisms (Table 1). In this paper, we will firstly discuss mechanisms of irisin's antioxidant action, then signaling pathways related to the antioxidant effect of irisin in the liver, and finally the conclusion of this review.

\section{Mechanisms of Irisin's Antioxidant Action}

The reduction in ROS production is due to the antioxidant effect of irisin. Understanding the approach of ROS 


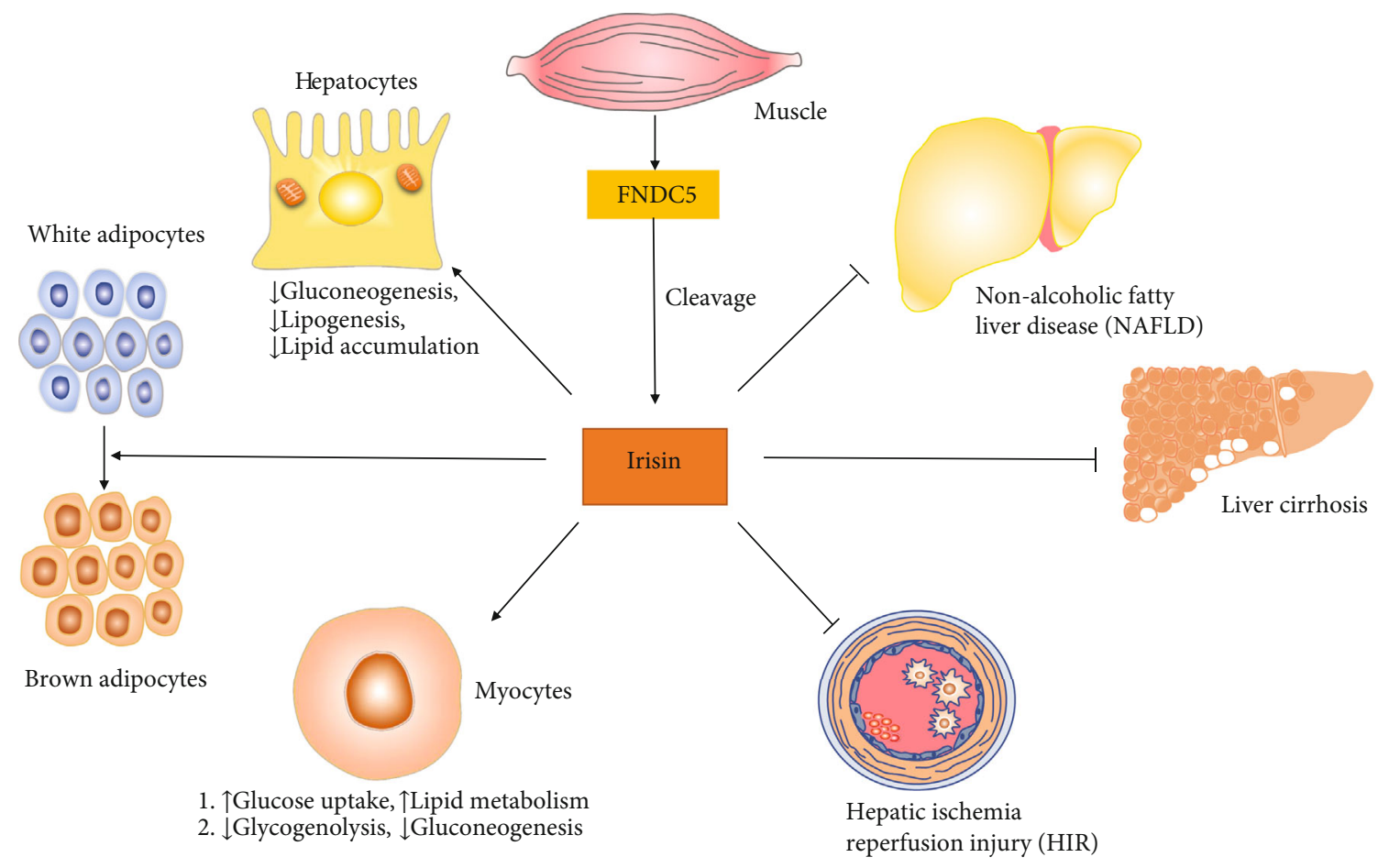

Figure 1: Pleiotropic traits of irisin.

production is helpful to comprehend the antioxidant mechanism of irisin. Free radicals are unstable and highly reactive atoms or molecules with unpaired electrons. There are two types of free radicals, reactive oxygen species (ROS) and reactive nitrogen species (RNS). ROS can be generated by intracellular and extracellular sources. Mitochondria are a major intracellular source for the production of ROS. In mitochondria, during the processes of electron transport to $\mathrm{O} 2$, variable degrees of reactive intermediates are formed, including ROS. Some redox-related enzymes (NO synthetase, xanthine oxidase, etc.) also produce ROS. In addition, macrophages and other monocytes contribute to the manufacture of ROS. The extracellular sources of ROS are mainly radiation, chemicals, and some virus infection [11]. Indeed, ROS are involved in a series of physiological processes, including signal transduction, microorganism eradication, and programmed cell death, all of which are essential for homeostasis stabilization. However, in most situations, high levels of ROS can oxidize all molecules in the cell membrane and contents, causing severe gene damage, and eventually triggering cell death by an apoptotic mechanism [12]. Unfortunately, the liver is the main attack target of free radicals. Intriguingly, we observed that, in hepatic ischemia reperfusion injury, hepatocytes express less antioxidant enzymes (superoxide dismutase (SOD) and glutathione $(\mathrm{GSH}))[13,14]$.

The antioxidant mechanism of irisin, in a broad sense, means not only the reduction of ROS but the decrease the consequential complications. The current literature showed that the antioxidant effect of irisin is associated with regulating several vital cellular processes, including modulating mitochondrial fission and fusion, inhibiting inflammasome activation, improving autophagy, suppressing ER stress and ferroptosis, and reversing cell death. Among the above cellular processes, modulating mitochondrial fission and fusion as well as improving autophagy is involved in reducing ROS production, whereas the processes such as inflammasome activation, ER stress, ferroptosis, and cell death are all the consequential complications of ROS damage. These ROS damage complications would paradoxically enhance the ROS production provided they are not eliminated [15]. In other words, controlling complications of ROS damage plays a dominating role in addressing oxidative stress.

2.1. Irisin Improves Mitochondrial Dynamics. Mitochondrial fission and fusion participate in regulating mitochondrial function and are controlled by mitochondrial fusion and fission-related genes, including Mitofusin1, 2 (Mfn1, 2), Dynamin-related protein 1 (Drp1), and Mitochondrial fission 1 protein (Fis1) [14]. Mitochondrial biogenesis (TFAM and PGC-1a gene controlled) is associated with selective inheritance, and functional mitochondrial biogenesis would limit the transmission of damaged mitochondrial genetic materials [16]. The balance between mitochondrial fission and fusion plays an important role in the maintenance of normal mitochondrial function. Previous research has shown that abnormal mitochondrial fission or fusion was a predictor of some diseases that are associated with oxidative stress and mitochondrial damage. Wu et al. suggested that quantum dots (QDs) might cause mitochondrial damage and overproduction of ROS by inducing imbalanced mitochondrial fission/fusion in HepG2 cells [17]. On the contrary, another study found that the upregulation of mitochondrial fusion led to less ROS production, which 
TABLE 1: The main mechanism of antioxidant effects of irisin.

\begin{tabular}{|c|c|c|c|c|}
\hline Study & $\begin{array}{l}\text { Crucial } \\
\text { molecules }\end{array}$ & $\begin{array}{c}\text { Liver } \\
\text { disease }^{1}\end{array}$ & Animal models & Results \\
\hline Bi et al. [14], 2019 & UCP-2 & HIR & $\mathrm{C} 7 \mathrm{BL} / 6 \mathrm{~J}$ mice $^{2}$ & $\begin{array}{l}\text { Irisin could suppress the production of ROS via upregulating } \\
\text { UCP- } 2 \text {. }\end{array}$ \\
\hline Bi et al. [14], 2019 & Drp-1, Fis-1 & HIR & C57BL/6J mice & $\begin{array}{l}\text { Irisin could decrease the expression of Drp-1 and Fis- } 1 \text { to inhibit } \\
\text { inappropriate mitochondrial fission for less oxidative stress. }\end{array}$ \\
\hline Bi et al.[14], 2019 & $\begin{array}{l}\text { PGC-1a, } \\
\text { TFAM }\end{array}$ & HIR & C57BL/6J mice & $\begin{array}{c}\text { Irisin might increase the level of the PGC-1a and TFAM to } \\
\text { decrease the oxidative stress by promoting mitochondrial } \\
\text { biogenesis. }\end{array}$ \\
\hline $\begin{array}{l}\text { Park et al. [62], } \\
2015\end{array}$ & PRMT3 & NAFLD & $\begin{array}{l}\text { AML12 cells }{ }^{3} / \text { mouse } \\
\text { primary hepatocytes }\end{array}$ & $\begin{array}{l}\text { Irisin may attenuate the function of PRMT3 to decrease the } \\
\text { production of ROS. }\end{array}$ \\
\hline $\begin{array}{l}\text { Fan et al. [27], } \\
2019\end{array}$ & $\begin{array}{l}\text { NLRP3 } \\
\text { inflammasomes }\end{array}$ & $\begin{array}{l}\text { Liver } \\
\text { fibrosis }\end{array}$ & $\begin{array}{l}\text { Adult male Sprague- } \\
\text { Dawley rats }\end{array}$ & $\begin{array}{l}\text { Irisin may inhibit the formation of the NLRP3 inflammasomes } \\
\text { to reduce the hepatic injury due to oxidative stress. }\end{array}$ \\
\hline Bi et al. [13], 2020 & $\begin{array}{l}\text { JNK, } \\
\text { telomerase }\end{array}$ & HIR & $\begin{array}{l}\text { Male Sprague-Dawley } \\
\text { rats }\end{array}$ & $\begin{array}{l}\text { Irisin would elevate the function of telomerase to promote the } \\
\text { autophagy of hepatocyte to reduce the production of ROS via } \\
\text { inhibiting JNK phosphorylation. }\end{array}$ \\
\hline $\begin{array}{l}\text { Zhang et al. [36], } \\
2020\end{array}$ & $\begin{array}{l}\text { Kindlin-2, } \alpha_{\mathrm{v}} \\
\text { integrins }\end{array}$ & HIR & C57BL/6J mice & $\begin{array}{l}\text { Kindlin- } 2 \text { may participate in the antioxidant effects of irisin. } \\
\text { The } \alpha_{\mathrm{v}} \text { integrins are transmembrane receptor of irisin to exert } \\
\text { irisin's antioxidant effect. }\end{array}$ \\
\hline $\begin{array}{l}\text { Mazur-Bialy and } \\
\text { Pocheć [71], } 2021\end{array}$ & Nrf2, HO-1 & NAFLD & $\begin{array}{l}\text { Quiescent macrophages/ } \\
\text { LPS-stimulated } \\
\text { macrophages }\end{array}$ & Irisin reduces oxidative stress via $\mathrm{Nrf} 2 / \mathrm{HO}-1$ involved pathway. \\
\hline Li et al. [89], 2021 & SIRT2 & NAFLD & C57BL/6J mice & $\begin{array}{c}\text { SIRT2 might maintain the stability of irisin to perform } \\
\text { antioxidant function. }\end{array}$ \\
\hline $\begin{array}{l}\text { Wei et al. [86], } \\
2020\end{array}$ & GPX4 & $\begin{array}{l}\text { Liver in } \\
\text { sepsis }\end{array}$ & C57BL/6J mice & $\begin{array}{c}\text { GPX4 is involved in the antioxidant effect of irisin and mitigates } \\
\text { the ferroptosis. }\end{array}$ \\
\hline
\end{tabular}

${ }^{1}$ The liver disease models established in the experiment or involved in relevant pathways. ${ }^{2} \mathrm{C} 57 \mathrm{BL} / 6 \mathrm{~J}$ mice is the most used inbred strain of laboratory mouse. ${ }^{3}$ AML12 cells were established from hepatocytes from a transgenic mouse with human TGF- $\alpha$. ROS: reactive oxygen species; NAFLD: nonalcoholic fatty liver disease; HIR: hepatic ischemia reperfusion injury; SOD: superoxide dismutase; UCP-2: Uncoupling protein-2; Drp1: Dynamin-related protein 1; Fis1: fission protein 1; PGC-1a: peroxisome proliferator-activated receptor gamma coactivator-1 alpha; TFAM: mitochondrial transcription factor A; PRMTs: protein arginine methyltransferases; NLRP3: nucleotide-binding oligomerization domain-like receptor 3; TERT: telomerase reverse transcriptase; JNK: c-Jun NH2terminal kinase; Nrf2: nuclear factor erythroid 2-related factor 2; HO-1: heme oxygenase 1; LPS: lipopolysaccharide; GPX-4: glutathione peroxidase-4; SIRT2: Sirtuin 2.

further validated the importance of mitochondrial fission/ fusion balance [18]. However, enhanced mitochondrial biogenesis promotes hepatocyte epithelial mesenchymal transition [19]. What is more, the restoration of mitochondrial dynamics participated in the suppression of oxidative stress. Das et al. demonstrated that melatonin helped to decrease ROS production by reducing abnormal mitochondrial dynamics [20]. Irisin modulates mitochondrial fusion, fission, and biogenesis to suppress oxidative stress. We found that irisin improved mitochondrial condition, exhibiting increased numbers of mitochondria and reduced mitochondrial fission or fusion. And irisin increased mitochondrial biogenesis [14]. Furthermore, we observed irisin significantly decreased ROS levels and decrease the inflammatory cytokines (ALT, AST, LDH) after liver ischemiareperfusion injury, which may be strongly associated with mitochondrial function improvement [14].

2.2. Irisin Downregulates Inflammasome. The inflammasome is recognized as one of the culprits of the oxidative stress damage [21]. In general, inflammasomes can mediate host immune responses to bacterial and cellular damage as forms of cytoplasmic protein complexes [21]. However, in patholog- ical condition, inflammasome activation is a severe complication of oxidative stress and could cause cell death and tissue damage. Especially, the NLRP3 inflammasome can trigger the activation of caspase- 1 to convert the immature cytokines pro-IL-1b and pro-IL-18 into mature cytokines IL-1b and IL18, respectively, to cause inflammatory damage. In addition, inflammasome complex activation is associated with liver inflammation and fibrosis. Shi et al. found that, in diabetic C57BL/6 mice, ROS-mediated NLRP3 inflammasome activation induced massive hepatocyte pyroptosis [22]. And it is also reported that carnitine palmitoyltransferase 1a (Cpt1a) would exacerbate ROS-induced inflammatory damage via the inflammasome activation signaling pathway in liver injury, which further demonstrated the tight relation between inflammasome activation and ROS damage [23]. The downregulation of inflammasome activation can mitigate ROS-induced inflammatory damage, exemplifying by a study in which allicin could reduce inflammasome activation to alleviate liver injury by suppressing hepatic oxidative stress [24]. Interestingly, another research discovered that NLRP3 inflammasome activation was downregulated when the ROS level was reduced by ginsenoside Rg1 in alcoholic hepatitis, implying the interaction between inflammasome activation and oxidative stress 
[25]. Moreover, Dong et al. indicated that the inhibition of inflammasome activation relied on the ROS-related pathway in nonalcoholic steatohepatitis [26]. Inflammasome activation is a major target of irisin to ameliorate oxidative damage in liver disease. Irisin inhibits inflammasome activation to protect against LPS-induced liver injury by reducing the ROS signaling pathway [15]. Fan et al. suggested that irisin participated in the hepatoprotection of dexmedetomidine by both suppressing oxidative stress and inhibiting inflammasome activation [27]. The irisin antibody partly abrogated the hepatoprotection of dexmedetomidine, which further confirmed the function of irisin [27]. Although the definite mechanism is unknown, it is hypothesized that dexmedetomidine promotes irisin to be grabbed from other body tissues, to be poured into blood circulation, and then to be transferred into liver tissue by dexmedetomidine [27]. Therefore, irisin level would be elevated in both serum and liver. Some studies found that, in cerebral ischemia mice, the low irisin level may be as a result of low-level physical exercise (brain damage-induced) $[27,28]$.

2.3. Irisin Decreases ER Stress. The endoplasmic reticulum (ER) is an essential organelle, possessing the majority of calcium and controlling protein translation [29]. The ER will respond to any disturbance of its normal function as well as the related signaling pathway, which is called ER stress [29]. And ER stress is associated with ROS level. A research found that both ER stress and ROS levels were increased in visfatin-treated livers and accompanied by hepatic damage [30]. Sharma et al. found that in hepatic stellate cells, ER stress and oxidative stress were induced by sorafenib to cause hepatic cell death, giving an evidence that ER stress was consistent with oxidative stress to cause liver damage together [31]. Previous research showed that decreasing ER stress levels was related to the inhibition of ROS production. An et al. indicated that black ginseng reduced ROS production in NAFLD through an ER stress-related pathway [32]. And another research observed that rosmarinic acid alleviated acrylamide-induced hepatic oxidative stress and decreased ER stress, which also implied that ER stress was a potential modulator of ROS production [33]. Irisin can regulate ER stress to attenuate hepatic oxidative stress. We found that irisin could significantly alleviate oxidative stress and ER stress in mice with acute pancreatitis [34]. Irisin may attenuate pancreatic fibrosis by reducing oxidative and ER stress [35]. In mice with liver steatosis, irisin also improves liver function and decreases ER stress and ROS production [36]. The irisin exerts a consistent function in oxidative stress and ER stress, indicating that ER stress is, at least in part, associated with antioxidant mechanism of irisin.

2.4. Irisin Promotes Autophagy. Autophagy plays a crucial role in a wide variety of pathological processes, including oxidative stress [37]. The overproduction of ROS will cause mitochondrial dysfunction and lipid peroxidation to break cellular homeostasis, in which autophagy may be induced [37]. It is found that autophagy participated in the repair of ROS-induced oxidative proteins in LO2 cells [38]. Chang et al. found that the production of ROS was elevated in
diTFPP/C2-ceramide-treated cells with the activation of autophagy, proving autophagy is involved in the oxidative processes [39]. The induction of autophagy also helps to suppress oxidative stress. A recent scientific study suggested that dihydrokaempferol reduced the production of ROS to attenuate liver injury by promoting autophagy [40]. Moreover, it is reported that 5-O-demethylnobiletin enhanced autophagy to mitigate oxidative stress in $\mathrm{CCl}_{4}$-induced acute liver injury, which further evidences that autophagy could ameliorate the ROS production [41]. Autophagy is a target of irisin to exert antioxidant effects. Specifically, irisin enhances the activation of autophagy to reduce mitochondrial permeability transition and then to decrease ROS levels. Li et al. used INS-1 cells as experimental models, revealing that metformin could elevate irisin expression and autophagy function to suppress cell apoptosis [42]. We used male Sprague-Dawley rats as animal models, observing that irisin would increase telomerase activity, autophagy, and improve oxidative stress in rats HIR. However, the inhibition of autophagy attenuated the irisin's hepatoprotective/ antioxidant effects after rats HIR, further verifying the autophagy's vital role in antioxidant effect of irisin [13].

2.5. Irisin Decreases Cell Death. Cell death (apoptosis/ferropotosis) is the definitive result of oxidative stress. In the early stage of apoptosis, the cellular ROS level is increased [43]. The overproduction of ROS will cause cellular redox status imbalance and organelle damage, leading to cell death eventually [43]. And it is discovered that Moringa oleifera fruit could cause ROS-mediated apoptosis in human liver cancer HepG2 cells [44]. What is more, Wang et al. demonstrated that EPS364, a deep-sea bacterial exopolysaccharide, exhibited an antitumor trait by inducing hepatic cell death by upregulating ROS production, supporting the idea that oxidative stress could trigger cell death [45]. And the idea is likewise proofed by another research where diTFPP/C2-ceramide increased hepatic cell death by increasing ROS levels [39]. Evidence has shown that the inhibition of ROS production can alleviate abnormal cell death. Hussein and colleagues found that chlorogenic acid, quercetin, coenzyme Q10, and silymarin reduced hepatic cell death and decreased ROS production [46]. Furthermore, another scientific research reported that Kushenol C reduced cell death by downregulating ROS levels and upregulating antioxidant enzyme expression, giving a more direct evidence that less oxidative stress induced less cell death [47]. Irisin exhibited antioxidant effects by improving abnormal cell death. Li et al. demonstrated that irisin suppressed oxidative stress and decreased cell death in LPS-induced liver injury [15]. It is also reported that the upregulation of hepatic irisin negatively regulated cell death and oxidative stress [48]. We also found that irisin improved autophagy to attenuate liver damage by decreasing ROS production and related cell death [13].

\section{Molecular Basis of Irisin's Antioxidant Effect}

According to the current literature, irisin might alleviate the oxidative stress through above cellular processes modulation 


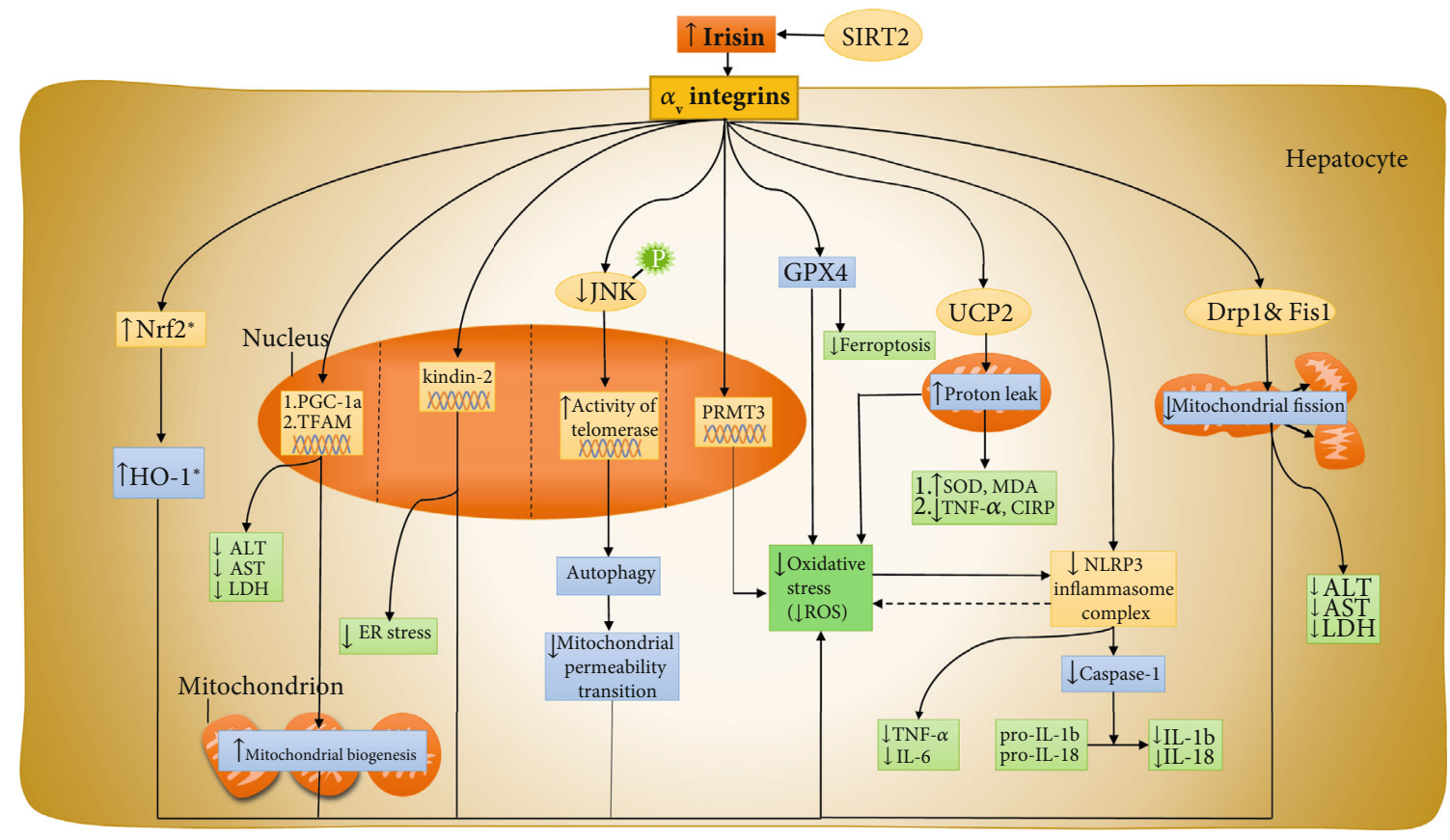

FIGURE 2: Signaling pathways of antioxidant effects of irisin in hepatocytes.

by regulating several intricate signaling pathways. And the core molecules in these signaling pathway are $\alpha_{\mathrm{v}}$ integrins, UCP-2, PRMT3, telomerase, Nrf2, Kindlin-2, JNK, GPX4, and SIRT2. Interpretation of the molecular basis of these signaling pathway is helpful to profoundly understand the mechanism of the antioxidant effect of irisin (Figure 2).

3.1. $\alpha_{v}$ Integrin Is the Transmembrane Receptor of Irisin. Integrins, a family of transmembrane cell adhesion molecules, take part in mediating cell-matrix and intercellular interactions [49]. $\alpha_{\mathrm{v}}$ integrins are pivots that connect fibroblasts, extracellular matrix, and monocytes [49]. Furthermore, in liver disease, $\alpha_{\mathrm{v}}$ integrins are crucial molecules in the development of liver fibrosis. And it is also reported that $\alpha_{\mathrm{v}}$ integrins regulated procollagen production in human hepatic stellate cells [50]. And a recent study likewise indicated that in $\alpha_{\mathrm{v}}$ integrin-knockout mice, the development of fibrosis was dampened, giving a further verification that $\alpha_{\mathrm{v}}$ integrins participated in the development of liver disease [51]. The inhibition of the expression of $\alpha_{\mathrm{v}}$ integrins will improve liver fibrosis. Zhang et al. suggested that the biphenyl compound IMB-S7 ameliorated liver fibrosis by downregulating $\alpha_{\mathrm{v}}$ integrin levels [52]. The $\alpha_{\mathrm{v}}$ integrins are also involved in the metastasis of cancer. Previous research demonstrated that fucoidan-Sargassum inhibited liver cancer metastasis by activating the $\alpha \mathrm{V} \beta 3 / \mathrm{Src} / \mathrm{E} 2 \mathrm{~F} 1$ signaling pathway [53]. Oxidative stress induces high levels of inflammatory cytokines, which affect the expression of $\alpha_{\mathrm{v}}$ integrins. It is reported that IL- 8 promoted the expression of $\alpha_{\mathrm{v}}$ integrins through the PI3K/Akt pathway in hepatocellular carcinoma [54]. The $\alpha_{\mathrm{v}}$ integrins, as an irisin transmembrane receptor, served the vital role in the antioxidant effect of irisin in liver disease. We found that irisin binds with $\alpha_{\mathrm{v}}$ integrins to suppress oxidative stress in mice with HIR injury [36,
55] (Figure 2). Although $\alpha_{\mathrm{v}}$ integrins are involved in the development of liver disease and participate in the antioxidant effect of irisin, there are still no direct evidences showing that the regulation of the $\alpha_{\mathrm{v}}$ integrins could affect the ROS production by irisin [36]. Many important questions about $\alpha_{\mathrm{v}}$ integrins, irisin, and their interaction remain unanswered and need further research.

3.2. Irisin Upregulates UCP-2. Mitochondrial proton leakage has a major impact on controlling ROS production [56]. Proton leakage allows a small amount of proton to flow back to the matrix to decrease the chemical gradients and electrical gradients [56]. Uncoupling protein-2 (UCP-2) belongs to the family of mitochondrial anion carrier proteins and is expressed in various tissues, including the liver [57]. UCP2 can decrease ROS emission from mitochondria by catalyzing proton leakage [58]. The uncoupling function and ROSeliminating mechanism of UCP-2 have been well studied. It is found that inhibition of UCP-2 causes a rapid increase in $\mathrm{H}_{2} \mathrm{O}_{2}$ production. In UCP-2 knockout mice, Arsenijevic et al. showed that the phagocytosis function of macrophages is enhanced due to the increased production of ROS in mitochondria [59]. Several studies have demonstrated the critical role of UCP-2 in irisin activity. Chen et al. showed that irisin stabilizes UCP-2 in the mitochondria and reduces its degradation, which in turn results in preservation of mitochondrial metabolism and protection from I/R-induced oxidative stress. We also found that irisin suppressed oxidative stress and upregulates UCP-2 expression after hepatic I/ $\mathrm{R}$ in $\mathrm{C} 57 \mathrm{BL} / 6 \mathrm{~J}$ mice [14] (Figure 2). In addition, we observed that irisin decreased the inflammatory cytokines (TNF- $\alpha$, CIRP) and increased the antioxidant enzymes level (SOD, MDA) [14]. The antioxidative effect of irisin was significantly attenuated after the administration of genipin (an inhibitor 
of UCP2), which demonstrates that UCP-2 is essential for the antioxidative activity of irisin [14].

3.3. Irisin Attenuates the Function of PRMT3. We found that liver oxidative injury was worse in HFD-induced, high serum fatty acid mice than in control group mice, suggesting that lipid contents fueled oxidative stress and that the inhibition of lipogenesis may play an important role in the antioxidant effect of irisin [14]. A recent study by Hoekstra and colleagues showed that the inhibition of protein arginine methyltransferases (PRMTs) holds down hepatic lipogenesis [60]. Moreover, previous research found that PRMT3 activation could be an inducer of fatty liver by binding to liver $\mathrm{X}$ receptor $\alpha(\mathrm{LXR} \alpha)$, which further supported that PRMT3 participated in lipogenesis [61]. Park and colleagues found indirect evidence that PRMT3 participates in the antioxidant effect of irisin [62]. They observed that in palmitic acid(PA-) treated mice, ROS levels, and lipogenic mediators were high [62]. High ROS levels are always followed by excessive inflammatory cytokine levels. Intriguingly, irisin reduced the expression of PRMT3 and ROS production as well as lipogenesis in PA-induced hepatic steatosis; however, the overexpression of PRMT3 reversed the antilipogenesis and anti-inflammatory function of irisin [62] (Figure 2). Although there was no direct evidence indicating that irisin alleviated oxidative stress by downregulating PRMT3 expression, at least in part, PRMT3 was involved in the antioxidant effect of irisin because PRMT3 played an important role in suppressing inflammatory stress, the chain reaction of oxidative stress [62].

3.4. Irisin Elevates the Function of Telomerase. Telomerase is a key enzyme responsible for maintaining the length of telomeres [63]. The activity of telomerase is associated with carcinogenesis and oxidative stress. Decreasing telomerase activity is present in liver fibrosis and hepatocyte senescence; however, increasing telomerase activity is seen in liver cancer [64]. In cancer cells, upregulation of ROS suppresses telomerase activity. Antioxidants protected telomerase activity in normal cells but paradoxically inhibited telomerase activity in cancer cells; however, the underlying mechanism is still unknown. Similarly, a recent literature observed that NAC, an antioxidant, decreased ROS levels and decreased telomerase activity, inducing cancer cell death [65]. Telomerase also participates in the regulation of autophagy. It is reported that downregulation of telomerase activity impaired autophagy function, leading to the overproduction of ROS [66]. Moreover, Green et al. also demonstrated that telomerase participated in the cellular response to oxidative stress by regulating autophagy, giving a further verification about interaction between telomerase and autophagy [67]. Irisin suppresses oxidative stress in a telomerase/autophagy-dependent manner. We found that irisin decreased the production of ROS by restoring autophagy function by upregulating telomerase activity [13]. After the administration of the telomerase inhibitor BIBP1532, the antioxidative effect of irisin was dampened, further confirming telomerase function [13] (Figure 2).
3.5. Irisin Promotes Nrf2 Signaling. Nuclear factor-E2-related factor 2 (Nrf2) is a crucial regulator of cellular redox homeostasis [11]. The inhibition of Nrf2 signaling is associated with increased ROS production [11]. Luo et al. demonstrated that the overexpression of carnitine palmitoyltransferase 1 stimulated the production of ROS in liver injury by suppressing the Nrf2/HO-1 signaling pathway [23]. Conversely, the activation of $\mathrm{Nrf} 2$ can ameliorate oxidative stress. According to a recent study, chlorogenic acid, quercetin, coenzyme Q10, and silymarin could significantly lower ROS levels by upregulating Nrf2 expression and HO-1 activity [46]. Furthermore, Shi and colleagues suggested that pelargonidin increased the Nrf2 level and decreased ROS production in $\mathrm{CCl}_{4}$-induced liver fibrosis [68]. Another study also investigated that adropin protected hepatocytes from oxidative stress by enhancing Nrf2 activity in NASH, further verifying the Nrf2 can reduce oxidative stress and has potential to become dominating effector protein of irisin [69]. As for Nrf2 might play an important role in the antioxidant effect of irisin. Mazur-Bialy et al. discovered that irisin improved the neutralization of ROS in both quiescent macrophages and LPS-stimulated macrophages and that the scavenging of ROS was more effective in LPS-stimulated situations, which confirmed the antioxidant effect of irisin [70]. Furthermore, they demonstrated that irisin performed antioxidant and anti-inflammatory functions by increasing the production of Nrf2 and HO- 1 in mouse macrophages, suggesting the Nrf2 is associated with antioxidant effect of irisin [71] (Figure 2). However, the evidence directly reveals that the role of Nrf2 in the antioxidant is still scarce and whether $\mathrm{Nrf} 2$ is regulated by irisin and exact molecular mechanism in antioxidant processes need more literature to confirm in the future [71].

3.6. Irisin Inhibits JNK Signaling. The c-Jun amino terminal kinase (JNK) signaling pathway is activated by various cellular stresses and participates in the modulation of cell inflammation and oxidative stress [72]. Previous studies showed that the production of ROS was associated with the activation of the JNK signaling pathway. Heo et al. found the upregulation of ER stress and the production of ROS as well as the activation of the JNK signaling pathway in the livers of visfatin-administered mice [30]. Another study demonstrated that hyperuricemia induced the activation of the JNK signaling pathway and caused elevated levels of ROS, indicating that the production of ROS may occur through the JNK signaling pathway [73]. In contrast, Li and colleagues found that guavinoside B alleviated ROS-induced liver damage and reduced the expression of the p-JNK gene, suggesting that negative correlation between JNK expression and oxidative damage [74]. Moreover, it is reported that orientin might activate the JNK signaling pathway to reduce oxidative stress, which offer the direct evidence about the antioxidant effect of JNK signaling pathway [75]. On the other hand, a high level of ROS could induce cancerization though the JNK pathway. Correspondingly, it is found that the overproduction of ROS triggered cholangiocellular carcinogenesis via the JNK signaling pathway [76]. The JNK pathway may participate in the antioxidant effect of irisin. 
In fact, some investigators observed that irisin inactivated the JNK pathway in vitro [77]. We also found that irisin suppressed oxidative stress by upregulating the activity of telomerase by decreasing the phosphorylation of JNK after hepatic I/R injury [13]. We administered anisomycin, a JNK MAPK inhibitor, to hepatocytes and found that the expression of irisin was not affected but that the activity of telomerase was significantly decreased after hepatic I/R injury, which further confirmed the function of the JNK signaling pathway [13] (Figure 2).

3.7. Kindlin-2 Is Involved in Antioxidant Effects of Irisin. Kindlin-2 is a widely expressed and highly conserved integrin-binding protein that is vital for cell-matrix adhesion, cell migration, and signaling $[78,79]$. The kindlin family is associated with oxidative stress. Previous researches demonstrated that kindlins could suppress oxidative stress [80]. Furthermore, Guo et al. reported that the depletion of kindlin-2 elevated the production of ROS in human A549 NSCLC cells [81]. Given the role of kindlin-2 in oxidative stress, it could be hypothesized that irisin might control ROS levels by interacting with kindlin-2. Our study showed that irisin improved mitochondrial function, oxidative stress, and ER stress after I/R injury in high-fat diet(HFD-) fed mice [36]. Notably, kindlin-2 is a main downstream molecule of $\alpha \mathrm{V} \beta 5$ integrin, and $\alpha \mathrm{V} \beta 5$ integrin is a receptor of irisin. Considering that irisin combined with $\alpha \mathrm{V} \beta 5$ integrin alleviates oxidative stress and HIR damage, the possibility that kindlin-2 interacts with irisin to improve oxidative damage is increased [36]. The possibility was fueled when we observed that the inhibition of kindlin-2 by RNAi eradicated the protective effect of irisin on mitochondrial function, ER stress, and oxidative stress [36] (Figure 2). Furthermore, we found that the expression of kindlin-2 was not affected by irisin in HFD-fed mice [36]. In this sense, kindlin-2 collaborated with irisin, and both acted in concert to exert antioxidant effects [36].

3.8. GPX4 Participates in the Antioxidant Effect of Irisin. Ferroptosis is a newly discovered cell death that is involved in a variety of disease processes, including NAFLD and ALD [82]. Consequently, the inhibition of ferroptosis is a therapeutic strategy for liver disease. Considering that the excessive accumulation of lipid ROS and increased iron levels are triggers of ferroptosis, antioxidant activity by irisin may be beneficial in liver disease improvement [83]. Glutathione peroxidase 4 (GPX4) is a lipid protective enzyme that eradicates lipid peroxides and prevents the formation of lipid ROS [84]. Furthermore, Yang and colleagues demonstrated that GPX4 was an important upstream modulator of ferroptosis and that GPX4 deficiency enhanced ferroptosis [85]. Overall, the regulation of GPX4 affects ferroptotic activity to ameliorate liver damage. Moreover, we explored the regulatory mechanism of GPX4 by irisin in the livers of septic mice [86]. In our study, we found that GPX4 expression was decreased, but iron levels and ROS levels were elevated in LPS-induced septic mice, indicating ferroptosis occurrence [86] (Figure 2). However, irisin treatment elevated GPX4 expression, markedly decreased ROS production, and decreased iron levels, suggesting that irisin may exert antioxidant effects in a GPX4-dependent manner [86]. It must be noted that when RSL3 was administered to inhibit GPX4 function, the protective effect of irisin disappeared, which further verified that GXP4 was a downstream molecule of irisin and played an essential role in the antioxidant effect of irisin [86]. Intriguingly, Yang et al. found that irisin was a positive regulator of ferroptosis. Irisin decreased GXP4 expression and upregulated ROS production to eliminate cancer cells in pancreatic cancer [87]. The paradoxical results may be a result of disease and tissue differences [87]. Taken together, GXP4 is a target molecule of irisin and participates in oxidative regulation.

3.9. SIRT2 Maintains the Stability of Irisin. In the above paragraphs, we demonstrated several antioxidant mechanisms of irisin in liver disease. Obviously, if the serum irisin concentration was low, its antioxidant effect would be impaired in liver disease. Zhang et al. found a negative correlation between serum irisin concentration and triglyceride contents in the liver in obese persons. And the irisin level was also low in NAFLD patients [88], which suggested that low irisin levels were associated with less protective effects. Therefore, the maintenance of normal serum irisin concentrations would also be important for irisin to exert antioxidant effects in liver disease. Furthermore, $\mathrm{Li}$ and colleagues found that under lipid stress, the acetylation and ubiquitination of the Fndc5 protein were dramatically strengthened, which accelerated Fndc5 degradation and consequently decreased the serum irisin concentration [89]. Based on this finding, we hypothesized that deacetylation and deubiquitination of Fndc5 would reverse the irisin level reduction. This hypothesis was supported by NAD + -boosting treatment promoting the deacetylation and deubiquitination of Fndc5 and restoring the irisin concentration. Interestingly, the depletion of Sirtuin 2 (SIRT2) diminished the effect of NAD+ and reduced the irisin level, suggesting that SIRT2 was associated with the stability of serum irisin levels. It was speculated that SIRT2 was the deacetylase of Fndc5. Moreover, $\mathrm{Li}$ et al. found that the K127/131 and K185/187/189 sites of Fndc5 were essential for the deacetylation activity of SIRT2 [89] (Figure 2). Lantier and colleagues demonstrated that the depletion of SIRT2 would cause abnormal mitochondrial biosynthesis, which might negatively influence the antioxidant effect of irisin [90]. This further confirmed that the interaction between irisin and SIRT2 played an important role in irisin to exert antioxidant effects in liver disease [90].

3.10. Other Possible Molecular Targets. There are several different antioxidant mechanisms of irisin that have been discovered in other organs, which implies that irisin might use these mechanisms to alleviate oxidative stress in the liver. In diabetes mellitus, irisin reduces ROS production by inhibiting the PKC- $\beta / \mathrm{NADPH}$ oxidase pathway [91]. In cardiovascular diseases, it was reported that irisin elevated the intracellular calcium level and upregulated mitochondrial thermogenesis to preserve cardiac function [92]. Zhang et al. demonstrated that irisin improved the vascular 
condition by inhibiting ROS/p38 MAPK/NF- $\kappa$ B signaling pathway function [93]. In addition, Wang et al. found that irisin kept the heart from oxidative damage through a SOD2-dependent mitochondrial modulation mechanism. Evidence has shown that irisin protects myocardial cells against I/R-stimulated mitochondrial damage by quelling the mitochondrial permeability transition pore and sustaining the normal mitochondrial membrane potential. In kidney disease, $\mathrm{Wu}$ et al. found that irisin can mitigate kidney oxidative injury by activating the FNDC5/irisin-AMPKSirt1-PGC- $1 \alpha$ signaling pathway [94]. In addition, UCP4 can inhibit the production of ROS and sustain the normal level of calcium to avoid apoptosis, which might be a downstream molecule of irisin [95].

\section{Conclusions}

In light of the important role of oxidative stress in liver disease, irisin may be an excellent therapeutic strategy for liver disease treatment. Irisin can decrease ROS production and protect hepatocytes against oxidative stress via several mechanisms. Irisin upregulates autophagy while downregulating ER stress, inflammasome activation, and cell death. Maintaining mitochondrial fusion, fission, and biogenesis balance is also an antioxidant mechanism of irisin. Furthermore, the $\alpha_{\mathrm{v}}$ integrin, a transmembrane receptor of irisin, combined with irisin to regulate antioxidant signaling pathway. And the molecular basis of the protective effect of irisin is to upregulate UCP2, to attenuate the activation of PRMT3, to elevate the function of telomerase, to activate Nrf2/HO-1 pathway, and to inhibit JNK signaling. Kindlin-2 and GPX4 also participated in the antioxidant effect of irisin. And SIRT2 might maintain the stability of irisin to perform antioxidant function. Moreover, irisin reduced the ROSinduced inflammatory reaction, which is helpful to improve tissue damage. Although the antioxidant effects of irisin have been elucidated and proven by many animal experiments, a lack of clinical data restricts the implementation of this potential therapeutic strategy in clinical practice.

Furthermore, there are few studies clearly demonstrating how irisin regulates cellular oxidative stress in detail, which highlights the importance for more-precise and betterdesign basic studies to uncover the exact role of irisin in the oxidative stress. On the basis of the current data, it is premature to draw a conclusion that irisin would improve liver diseases outcomes in human body. More well-design prospective clinical studies are imperative to access the usage of irisin as a therapeutic approach in liver diseases, which should be the major research aim for the near future.

\section{Abbreviations}

ROS: $\quad$ Reactive oxygen species

RNS: $\quad$ Reactive nitrogen species

NAFLD: Nonalcoholic fatty liver disease

HIR: Hepatic ischemia reperfusion injury

FNDC5: Fibronectin type III domain containing 5

NASH: Nonalcoholic steatohepatitis

SOD: $\quad$ Superoxide dismutase
GSH: Glutathione

UCPs: Uncoupling proteins

UCP-2: Uncoupling protein-2

CIRP: Cold-inducible RNA binding protein

MDA: Malonaldehyde

Drp1: Dynamin-related protein 1

OMM: Outer mitochondrial membrane

Fis1: $\quad$ Fission protein 1

Mff: $\quad$ Mitochondrial fission factor

PGC-1a: Peroxisome proliferator-activated receptor gamma coactivator-1 alpha

TFAM: Mitochondrial transcription factor A

PRMTs: Protein arginine methyltransferases

PA: $\quad$ Palmitic acid

$\mathrm{NF} \kappa \mathrm{B}$ : Nuclear factor $\kappa \mathrm{B}$

NLRP3: Nucleotide-binding oligomerization domain-like receptor 3

Cptla: Carnitine palmitoyltransferase 1a

QDs: Quantum dots

JNK: C-Jun NH2-terminal kinase

MAPK: Mitogen-activated protein kinase

HFD: High-fat diet

ER: $\quad$ Endoplasmic reticulum

Nrf2: $\quad$ Nuclear factor erythroid 2-related factor 2

HO-1: Heme oxygenase 1

LPS: Lipopolysaccharide

iNOS: Inducible nitric oxide synthase

GPX-4: Glutathione peroxidase-4

CAT: Catalase

SIRT2: Sirtuin 2.

\section{Conflicts of Interest}

The authors declare no competing interests.

\section{Acknowledgments}

This work was supported by grants from the National Natural Science Foundation of China (No. 81770491) and the Innovation Capacity Support Plan of Shaanxi Province (No. 2020TD-040).

\section{References}

[1] P. Boström, J. Wu, M. P. Jedrychowski et al., "A PGC1- $\alpha-$ dependent myokine that drives brown-fat-like development of white fat and thermogenesis," Nature, vol. 481, no. 7382, pp. 463-468, 2012.

[2] J. Zhang, P. Valverde, X. Zhu et al., "Exercise-induced irisin in bone and systemic irisin administration reveal new regulatory mechanisms of bone metabolism," Bone Research, vol. 5, p. 16056, 2017.

[3] H. Li, F. Wang, M. Yang, J. Sun, Y. Zhao, and D. Tang, "The effect of irisin as a metabolic regulator and its therapeutic potential for obesity," International Journal of Endocrinology, vol. 2021, Article ID 6572342, 12 pages, 2021.

[4] S. Jiang, L. Piao, E. B. Ma, H. Ha, and J. Y. Huh, "Associations of circulating irisin with FNDC5 expression in fat and muscle in type 1 and type 2 diabetic mice," Biomolecules, vol. 11, no. 2, 2021. 
[5] C. Xin, J. Liu, J. Zhang et al., "Irisin improves fatty acid oxidation and glucose utilization in type 2 diabetes by regulating the AMPK signaling pathway," International Journal of Obesity, vol. 40, no. 3, pp. 443-451, 2016.

[6] T. Y. Liu, C. X. Shi, R. Gao et al., "Irisin inhibits hepatic gluconeogenesis and increases glycogen synthesis via the PI3K/Akt pathway in type 2 diabetic mice and hepatocytes," Clinical Science (London, England), vol. 129, no. 10, pp. 839-850, 2015.

[7] H. Tang, R. Yu, S. Liu, B. Huwatibieke, Z. Li, and W. Zhang, "Irisin inhibits hepatic cholesterol synthesis via AMPKSREBP2 signaling," eBioMedicine, vol. 6, pp. 139-148, 2016.

[8] M. R. Szabó, M. Pipicz, T. Csont, and C. Csonka, "Modulatory effect of myokines on reactive oxygen species in ischemia/ reperfusion," International journal of molecular sciences, vol. 21, no. 24, p. 9382, 2020.

[9] Z. Boyer-Diaz, P. Morata, P. Aristu-Zabalza, A. Gibert-Ramos, J. Bosch, and J. Gracia-Sancho, "Oxidative stress in chronic liver disease and portal hypertension: potential of DHA as nutraceutical," Nutrients, vol. 12, no. 9, 2020.

[10] H. Cichoż-Lach and A. Michalak, "Oxidative stress as a crucial factor in liver diseases," World Journal of Gastroenterology, vol. 20, no. 25, pp. 8082-8091, 2014.

[11] S. Sajadimajd and M. Khazaei, "Oxidative stress and cancer: the role of Nrf2," Current Cancer Drug Targets, vol. 18, no. 6, pp. 538-557, 2018.

[12] S. Yang and G. Lian, "ROS and diseases: role in metabolism and energy supply," Molecular and Cellular Biochemistry, vol. 467, no. 1-2, pp. 1-12, 2020.

[13] J. Bi, L. Yang, T. Wang et al., "Irisin improves autophagy of aged hepatocytes via increasing telomerase activity in liver injury," Oxidative Medicine and Cellular Longevity, vol. 2020, Article ID 6946037, 13 pages, 2020.

[14] J. Bi, J. Zhang, Y. Ren et al., "Irisin alleviates liver ischemiareperfusion injury by inhibiting excessive mitochondrial fission, promoting mitochondrial biogenesis and decreasing oxidative stress," Redox Biology, vol. 20, pp. 296-306, 2019.

[15] Q. Li, Y. Tan, S. Chen et al., "Irisin alleviates LPS-induced liver injury and inflammation through inhibition of NLRP3 inflammasome and NF- $\kappa \mathrm{B}$ signaling," Journal of Receptor and Signal Transduction Research, vol. 41, no. 3, pp. 294303, 2021.

[16] L. D. Popov, "Mitochondrial biogenesis: an update," Journal of Cellular and Molecular Medicine, vol. 24, no. 9, pp. 4892-4899, 2020.

[17] D. Wu, J. Lu, Y. Ma, Y. Cao, and T. Zhang, "Mitochondrial dynamics and mitophagy involved in MPA-capped CdTe quantum dots- induced toxicity in the human liver carcinoma (HepG2) cell line," Environmental Pollution, vol. 274, article 115681, 2021.

[18] C. Zhang, Y. Jia, B. Liu, G. Wang, and Y. Zhang, "TLR4 knockout upregulates the expression of Mfn2 and PGC- $1 \alpha$ in a highfat diet and ischemia-reperfusion mice model of liver injury," Life Sciences, vol. 254, article 117762, 2020.

[19] L. Frattaruolo, M. Brindisi, R. Curcio, F. Marra, V. Dolce, and A. R. Cappello, "Targeting the mitochondrial metabolic network: a promising strategy in cancer treatment," International journal of molecular sciences, vol. 21, no. 17, 2020.

[20] N. Das, A. Mandala, S. Naaz et al., "Melatonin protects against lipid-induced mitochondrial dysfunction in hepatocytes and inhibits stellate cell activation during hepatic fibrosis in mice," Journal of pineal research, vol. 62, no. 4, 2017.
[21] A. Abderrazak, T. Syrovets, D. Couchie et al., "NLRP3 inflammasome: from a danger signal sensor to a regulatory node of oxidative stress and inflammatory diseases," Redox Biology, vol. 4, pp. 296-307, 2015.

[22] C. Shi, Q. Wang, Z. Rao et al., "Diabetes induces hepatocyte pyroptosis by promoting oxidative stress-mediated NLRP3 inflammasome activation during liver ischaemia and reperfusion injury," Annals of Translational Medicine, vol. 8, no. 12, p. 739, 2020.

[23] X. Luo, D. Sun, Y. Wang, F. Zhang, and Y. Wang, "Cptla promoted ROS-induced oxidative stress and inflammation in liver injury via the Nrf2/HO-1 and NLRP 3 inflammasome signaling pathway," Canadian Journal of Physiology and Pharmacology, vol. 99, pp. 468-477, 2021.

[24] B. Nan, C. Yang, L. Li et al., "Allicin alleviated acrylamideinduced NLRP3 inflammasome activation via oxidative stress and endoplasmic reticulum stress in Kupffer cells and SD rats liver," Food and Chemical Toxicology, vol. 148, article 111937, 2021.

[25] C. Yang, X. He, J. Zhao, and W. Huang, "Hepatoprotection by Ginsenoside Rg1 in alcoholic liver disease," International Immunopharmacology, vol. 92, article 107327, 2021.

[26] Z. Dong, Q. Zhuang, X. Ye et al., “Adiponectin inhibits NLRP3 inflammasome activation in nonalcoholic steatohepatitis via AMPK-JNK/ErK1/2-NF $\kappa$ B/ROS signaling pathways," Frontiers in Medicine, vol. 7, article 546445, 2020.

[27] X. Fan, J. Du, M.-H. Wang et al., "Irisin contributes to the hepatoprotection of dexmedetomidine during intestinal ischemia/reperfusion," Oxidative Medicine and Cellular Longevity, vol. 2019, Article ID 7857082, 15 pages, 2019.

[28] D. J. Li, Y. H. Li, H. B. Yuan, L. F. Qu, and P. Wang, "The novel exercise-induced hormone irisin protects against neuronal injury via activation of the Akt and ERK1/2 signaling pathways and contributes to the neuroprotection of physical exercise in cerebral ischemia," Metabolism, Clinical and Experimental, vol. 68, pp. 31-42, 2017.

[29] S. A. Oakes and F. R. Papa, "The role of endoplasmic reticulum stress in human pathology," Annual Review of Pathology, vol. 10, pp. 173-194, 2015.

[30] Y. J. Heo, S. E. Choi, N. Lee et al., "Visfatin exacerbates hepatic inflammation and fibrosis in a methionine-choline-deficient diet mouse model," Journal of Gastroenterology and Hepatology, vol. 36, no. 9, pp. 2592-2600, 2021.

[31] S. Sharma, S. M. Ghufran, S. Ghose, and S. Biswas, "Cytoplasmic vacuolation with endoplasmic reticulum stress directs sorafenib induced non-apoptotic cell death in hepatic stellate cells," Scientific Reports, vol. 11, no. 1, p. 3089, 2021.

[32] M. Y. An, S. R. Lee, H. J. Hwang, J. G. Yoon, H. J. Lee, and J. A. Cho, "Antioxidant and anti-inflammatory effects of Korean black ginseng extract through ER stress pathway," Antioxidants (Basel, Switzerland), vol. 10, no. 1, 2021.

[33] Z. Hong, W. Minghua, N. Bo et al., "Rosmarinic acid attenuates acrylamide induced apoptosis of BRL-3A cells by inhibiting oxidative stress and endoplasmic reticulum stress," Food and Chemical Toxicology, vol. 151, article 112156, 2021.

[34] Y. F. Ren, M. Z. Wang, J. B. Bi et al., "Irisin attenuates intestinal injury, oxidative and endoplasmic reticulum stress in mice with L-arginine-induced acute pancreatitis," World Journal of Gastroenterology, vol. 25, no. 45, pp. 6653-6667, 2019.

[35] Y. Ren, J. Zhang, M. Wang et al., "Identification of irisin as a therapeutic agent that inhibits oxidative stress and fibrosis in 
a murine model of chronic pancreatitis," Biomedicine \& pharmacotherapy = Biomedecine \& pharmacotherapie, vol. 126, article 110101, 2020.

[36] J. Zhang, Y. Ren, J. Bi et al., "Involvement of kindlin-2 in irisin's protection against ischaemia reperfusion-induced liver injury in high-fat diet-fed mice," Journal of Cellular and Molecular Medicine, vol. 24, no. 22, pp. 13081-13092, 2020.

[37] H. R. Yun, Y. H. Jo, J. Kim, Y. Shin, S. S. Kim, and T. G. Choi, "Roles of autophagy in oxidative stress," International journal of molecular sciences, vol. 21, no. 9, p. 3289, 2020.

[38] Y. Wang, L. Xu, L. Peng et al., "Polybrominated diphenyl ethers quinone-induced intracellular protein oxidative damage triggers ubiquitin-proteasome and autophagy-lysosomal system activation in LO2 cells," Chemosphere, vol. 275, article 130034, 2021.

[39] W. T. Chang, Y. D. Bow, Y. C. Chen et al., "The phenoxyphenol compound diTFPP mediates exogenous C(2)-ceramide metabolism, inducing cell apoptosis accompanied by ROS formation and autophagy in hepatocellular carcinoma cells," Antioxidants (Basel, Switzerland), vol. 10, no. 3, 2021.

[40] J. Zhang, C. Hu, X. Li et al., "Protective effect of dihydrokaempferol on acetaminophen-induced liver injury by activating the SIRT1 pathway," The American Journal of Chinese Medicine, vol. 49, no. 3, pp. 705-718, 2021.

[41] S. N. Chang, S. H. Kim, D. K. Dey et al., "5-O-Demethylnobiletin alleviates CCl4-Induced acute liver injury by equilibrating ROS-mediated apoptosis and autophagy induction," International journal of molecular sciences, vol. 22, no. 3, p. 1083, 2021.

[42] Q. Li, S. Jia, L. Xu, B. Li, and N. Chen, "Metformin-induced autophagy and irisin improves INS-1 cell function and survival in high-glucose environment via AMPK/SIRT1/PGC- $1 \alpha$ signal pathway," Food Science \& Nutrition, vol. 7, no. 5, pp. 1695-1703, 2019.

[43] Y. Xie, W. Hou, X. Song et al., "Ferroptosis: process and function," Cell Death and Differentiation, vol. 23, no. 3, pp. 369379, 2016.

[44] S. Siddiqui, S. Upadhyay, I. Ahmad, A. Hussain, and M. Ahamed, "Cytotoxicity of Moringa oleifera fruits on human liver cancer and molecular docking analysis of bioactive constituents against caspase-3 enzyme," Journal of Food Biochemistry, vol. 45, article e13720, 2021.

[45] Y. Wang, G. Liu, R. Liu, M. Wei, J. Zhang, and C. Sun, "EPS364, a novel deep-sea bacterial exopolysaccharide, inhibits liver cancer cell growth and adhesion," Marine drugs, vol. 19, no. 3, 2021.

[46] R. M. Hussein, D. M. Sawy, M. A. Kandeil, and H. S. Farghaly, "Chlorogenic acid, quercetin, coenzyme Q10 and silymarin modulate Keap1-Nrf2/heme oxygenase-1 signaling in thioacetamide-induced acute liver toxicity," Life Sciences, vol. 277, article 119460, 2021.

[47] B. O. Cho, J. H. Kim, D. N. Che et al., "Kushenol C prevents tert-Butyl hydroperoxide and acetaminophen-induced liver injury," Molecules, vol. 26, no. 6, 2021.

[48] C. M. Canivet, S. Bonnafous, D. Rousseau et al., "Hepatic FNDC5 is a potential local protective factor against nonalcoholic fatty liver," Biochimica et biophysica acta Molecular basis of disease, vol. 1866, no. 5, article 165705, 2020.

[49] I. D. Campbell and M. J. Humphries, "Integrin structure, activation, and interactions," Cold Spring Harbor perspectives in biology, vol. 3, no. 3, 2011.
[50] Z. Han, Y. Ma, G. Cao et al., "Integrin $\alpha \mathrm{V} \beta 1$ regulates procollagen I production through a non-canonical transforming growth factor $\beta$ signaling pathway in human hepatic stellate cells," The Biochemical Journal, vol. 478, no. 9, pp. 16891703, 2021.

[51] N. I. Reed, H. Jo, C. Chen et al., "The $\alpha v \beta 1$ integrin plays a critical in vivo role in tissue fibrosis," Science Translational Medicine, vol. 7, no. 288, article 288ra79, 2015.

[52] N. Zhang, S. S. Zhao, Y. X. Zhang et al., "A novel biphenyl compound IMB-S7 ameliorates hepatic fibrosis in BDL rats by suppressing Sp1-mediated integrin $\alpha \mathrm{v}$ expression," Acta Pharmacologica Sinica, vol. 41, no. 5, pp. 661-669, 2020.

[53] T. J. Pan, L. X. Li, J. W. Zhang et al., "Antimetastatic effect of Fucoidan-Sargassum against liver cancer cell invadopodia formation via targeting integrin $\alpha \mathrm{V} \beta 3$ and mediating $\alpha \mathrm{V} \beta 3 / \mathrm{Src} /$ E2F1 signaling," Journal of Cancer, vol. 10, no. 20, pp. 47774792, 2019.

[54] F. Sun, J. Wang, Q. Sun et al., "Interleukin-8 promotes integrin $\beta 3$ upregulation and cell invasion through PI3K/Akt pathway in hepatocellular carcinoma," Journal of Experimental \& Clinical Cancer Research, vol. 38, no. 1, p. 449, 2019.

[55] H. Kim, C. D. Wrann, M. Jedrychowski et al., "Irisin mediates effects on bone and fat via $\alpha \mathrm{V}$ integrin receptors," Cell, vol. 175, no. 7, pp. 1756-1768.e17, 2018.

[56] S. Cadenas, "Mitochondrial uncoupling, ROS generation and cardioprotection," Biochimica et Biophysica Acta - Bioenergetics, vol. 1859, no. 9, pp. 940-950, 2018.

[57] P. Ježek, B. Holendová, K. D. Garlid, and M. Jabůrek, "Mitochondrial uncoupling proteins: subtle regulators of cellular redox signaling," Antioxidants \& Redox Signaling, vol. 29, no. 7, pp. 667-714, 2018.

[58] L. J. Toime and M. D. Brand, "Uncoupling protein-3 lowers reactive oxygen species production in isolated mitochondria," Free Radical Biology \& Medicine, vol. 49, no. 4, pp. 606-611, 2010.

[59] D. Arsenijevic, H. Onuma, C. Pecqueur et al., "Disruption of the uncoupling protein-2 gene in mice reveals a role in immunity and reactive oxygen species production," Nature Genetics, vol. 26, no. 4, pp. 435-439, 2000.

[60] M. Hoekstra, J. E. Nahon, L. M. de Jong, M. J. Kröner, L. R. de Leeuw, and M. Van Eck, "Inhibition of PRMT3 activity reduces hepatic steatosis without altering atherosclerosis susceptibility in apoE knockout mice," Biochimica et biophysica acta," Molecular basis of disease, vol. 1865, no. 6, pp. 14021409, 2019.

[61] D. I. Kim, M. J. Park, S. K. Lim et al., "PRMT3 regulates hepatic lipogenesis through direct interaction with $\operatorname{LXR} \alpha$," Diabetes, vol. 64 , no. 1, pp. 60-71, 2015.

[62] M.-J. Park, D.-I. Kim, J.-H. Choi, Y.-R. Heo, and S.-H. Park, "New role of irisin in hepatocytes: the protective effect of hepatic steatosis in vitro," Cellular Signalling, vol. 27, no. 9, pp. 1831-1839, 2015.

[63] Y. Wang, L. Sušac, and J. Feigon, "Structural biology of telomerase," Cold Spring Harbor perspectives in biology, vol. 11, no. 12, 2019.

[64] J. C. Nault, M. Ningarhari, S. Rebouissou, and J. ZucmanRossi, "The role of telomeres and telomerase in cirrhosis and liver cancer," Nature Reviews. Gastroenterology \& Hepatology, vol. 16, no. 9, pp. 544-558, 2019.

[65] P. Li, M. Wu, J. Wang, Y. Sui, S. Liu, and D. Shi, "NAC selectively inhibit cancer telomerase activity: a higher redox 
homeostasis threshold exists in cancer cells," Redox Biology, vol. 8, pp. 91-97, 2016.

[66] X. Ding, Z. Nie, Z. She et al., "The regulation of ROS- and BECN1-mediated autophagy by human telomerase reverse transcriptase in glioblastoma," Oxidative Medicine and Cellular Longevity, vol. 2021, Article ID 6636510, 10 pages, 2021.

[67] P. D. Green, N. K. Sharma, and J. H. Santos, “Telomerase impinges on the cellular response to oxidative stress through mitochondrial ROS-mediated regulation of autophagy," International journal of molecular sciences, vol. 20, no. 6, 2019.

[68] Y. S. Shi, X. X. Li, H. T. Li, and Y. Zhang, "Pelargonidin ameliorates $\mathrm{CCl}(4)$-induced liver fibrosis by suppressing the ROSNLRP3-IL-1 $\beta$ axis via activating the Nrf2 pathway," Food \& Function, vol. 11, no. 6, pp. 5156-5165, 2020.

[69] X. Chen, H. Xue, W. Fang et al., “Adropin protects against liver injury in nonalcoholic steatohepatitis via the Nrf2 mediated antioxidant capacity," Redox Biology, vol. 21, article 101068, 2019.

[70] A. I. Mazur-Bialy, K. Kozlowska, E. Pochec, J. Bilski, and T. Brzozowski, "Myokine irisin-induced protection against oxidative stress in vitro. Involvement of heme oxygenase-1 and antioxidazing enzymes superoxide dismutase-2 and glutathione peroxidase," Journal of physiology and pharmacology: an official journal of the Polish Physiological Society, vol. 69, no. 1, pp. 117-125, 2018.

[71] A. I. Mazur-Bialy and E. Pocheć, "The time-course of antioxidant irisin activity: role of the Nrf2/HO-1/HMGB1 axis," Antioxidants (Basel, Switzerland), vol. 10, no. 1, 2021.

[72] Q. Wu, W. Wu, B. Fu, L. Shi, X. Wang, and K. Kuca, "JNK signaling in cancer cell survival," Medicinal Research Reviews, vol. 39, no. 6, pp. 2082-2104, 2019.

[73] D. Xie, H. Zhao, J. Lu et al., "High uric acid induces liver fat accumulation via ROS/JNK/AP-1 signaling," American Journal of Physiology. Endocrinology and Metabolism, vol. 320, no. 6, pp. E1032-E1043, 2021.

[74] Y. Li, J. Xu, D. Li et al., "Guavinoside B from Psidium guajava alleviates acetaminophen-induced liver injury via regulating the Nrf2 and JNK signaling pathways," Food \& Function, vol. 11, no. 9, pp. 8297-8308, 2020.

[75] Q. Xiao, R. Piao, H. Wang, C. Li, and L. Song, "Orientin-mediated $\mathrm{Nrf} 2 / \mathrm{HO}-1$ signal alleviates $\mathrm{H}(2) \mathrm{O}(2)$-induced oxidative damage via induction of JNK and PI3K/AKT activation," International Journal of Biological Macromolecules, vol. 118, no. Part A, pp. 747-755, 2018.

[76] D. Yuan, S. Huang, E. Berger et al., "Kupffer cell-derived Tnf triggers cholangiocellular tumorigenesis through JNK due to chronic mitochondrial dysfunction and ROS," Cancer Cell, vol. 31, no. 6, pp. 771-789.e6, 2017.

[77] G. Vadalà, G. Di Giacomo, L. Ambrosio et al., "Irisin recovers osteoarthritic chondrocytes in vitro," Cells, vol. 9, no. 6, 2020.

[78] E. Rognoni, R. Ruppert, and R. Fässler, "The kindlin family: functions, signaling properties and implications for human disease," Journal of Cell Science, vol. 129, no. 1, pp. 17-27, 2016.

[79] L. Guo, T. Cai, K. Chen et al., "Kindlin-2 regulates mesenchymal stem cell differentiation through control of YAP1/TAZ," The Journal of Cell Biology, vol. 217, no. 4, pp. 1431-1451, 2018.

[80] H. Emmert, J. Culley, and V. G. Brunton, "Inhibition of cyclindependent kinase activity exacerbates $\mathrm{H}(2) \mathrm{O}(2)$-induced
DNA damage in Kindler syndrome keratinocytes," Experimental Dermatology, vol. 28, no. 9, pp. 1074-1078, 2019.

[81] L. Guo, C. Cui, K. Zhang et al., "Kindlin-2 links mechanoenvironment to proline synthesis and tumor growth," Nature Communications, vol. 10, no. 1, p. 845, 2019.

[82] M. Jia, H. Zhang, Q. Qin et al., "Ferroptosis as a new therapeutic opportunity for nonviral liver disease," European Journal of Pharmacology, vol. 908, article 174319, 2021.

[83] B. R. Stockwell, J. P. Friedmann Angeli, H. Bayir et al., "Ferroptosis: a regulated cell death nexus linking metabolism, redox biology, and disease," Cell, vol. 171, no. 2, pp. 273-285, 2017.

[84] G. C. Forcina and S. J. Dixon, "GPX4 at the crossroads of lipid homeostasis and ferroptosis," Proteomics, vol. 19, no. 18, article e1800311, 2019.

[85] W. S. Yang, R. SriRamaratnam, M. E. Welsch et al., "Regulation of ferroptotic cancer cell death by GPX4," Cell, vol. 156, no. 1-2, pp. 317-331, 2014.

[86] S. Wei, J. Bi, L. Yang et al., "Serum irisin levels are decreased in patients with sepsis, and exogenous irisin suppresses ferroptosis in the liver of septic mice," Clinical and Translational Medicine, vol. 10, no. 5, article e173, 2020.

[87] B. C. Yang and P. S. Leung, "Irisin is a positive regulator for ferroptosis in pancreatic cancer," Molecular Therapy-Oncolytics, vol. 18, pp. 457-466, 2020.

[88] H. J. Zhang, X. F. Zhang, Z. M. Ma et al., "Irisin is inversely associated with intrahepatic triglyceride contents in obese adults," Journal of Hepatology, vol. 59, no. 3, pp. 557-562, 2013.

[89] D. J. Li, S. J. Sun, J. T. Fu et al., "NAD(+)-boosting therapy alleviates nonalcoholic fatty liver disease via stimulating a novel exerkine Fndc5/irisin," Theranostics, vol. 11, no. 9, pp. 43814402, 2021.

[90] L. Lantier, A. S. Williams, C. C. Hughey et al., "SIRT2 knockout exacerbates insulin resistance in high fat-fed mice," PLoS One, vol. 13, no. 12, article e0208634, 2018.

[91] D. Zhu, H. Wang, J. Zhang et al., "Irisin improves endothelial function in type 2 diabetes through reducing oxidative/nitrative stresses," Journal of Molecular and Cellular Cardiology, vol. 87, pp. 138-147, 2015.

[92] C. Xie, Y. Zhang, T. D. Tran et al., "Irisin controls growth, intracellular $\mathrm{Ca} 2+$ signals, and mitochondrial thermogenesis in cardiomyoblasts," PLoS One, vol. 10, no. 8, article e0136816, 2015.

[93] Y. Zhang, Q. Mu, Z. Zhou et al., "Protective effect of irisin on atherosclerosis via suppressing oxidized low density lipoprotein induced vascular inflammation and endothelial dysfunction," PLoS One, vol. 11, no. 6, article e0158038, 2016.

[94] F. Wu, Z. Li, M. Cai et al., "Aerobic exercise alleviates oxidative stress-induced apoptosis in kidneys of myocardial infarction mice by inhibiting ALCAT1 and activating FNDC5/Irisin signaling pathway," Free Radical Biology \& Medicine, vol. 158, pp. 171-180, 2020.

[95] M. P. Mattson and D. Liu, "Mitochondrial potassium channels and uncoupling proteins in synaptic plasticity and neuronal cell death," Biochemical and Biophysical Research Communications, vol. 304, no. 3, pp. 539-549, 2003. 